\title{
Restaurant-associated outbreak of Salmonella Typhi in Nauru: an epidemiological and cost analysis
}

\author{
S. J. OLSEN ${ }^{1,2 *}$, B. KAFOA ${ }^{3}$, N. S. S. WIN ${ }^{4}$, M. JOSE ${ }^{4}$, W. BIBB ${ }^{1}$, S. LUBY ${ }^{1}$, \\ G. WAIDUBU ${ }^{4}$, M. O' LEARY ${ }^{5}$ AND E. MINTZ ${ }^{1}$ \\ ${ }^{1}$ Foodborne and Diarrheal Diseases Branch, Division of Bacterial and Mycotic Diseases, National Center for \\ Infectious Diseases, Centers for Disease Control and Prevention (CDC), 1600 Clifton Road, Mailstop A-38, \\ Atlanta GA 30333, USA \\ ${ }^{2}$ Epidemic Intelligence Service, Division of Applied Public Health Training, Epidemiology Program Office, \\ $C D C$ \\ ${ }^{3}$ Fiji School of Medicine, Suva, Fiji \\ ${ }^{4}$ Nauru General Hospital, Nauru \\ ${ }^{5}$ Western Pacific Region of the World Health Organization, Suva, Fiji
}

(Accepted 15 June 2001)

\section{SUMMARY}

Typhoid fever is endemic in the South Pacific. We investigated an outbreak in Nauru. Through interviews and medical records, we identified 50 persons with onset between 1 October 1998 and 10 May 1999, of fever lasting $\geqslant 3$ days and one other symptom. Salmonella Typhi was isolated from $19(38 \%)$ cases. Thirty-two $(64 \%)$ patients were school-aged children, and 17 $(34 \%)$ were in four households. Case-control studies of (a) culture-confirmed cases and ageand neighbourhood-matched controls; and (b) household index cases and randomly selected age-matched controls implicated two restaurants: Restaurant M (matched OR $[\mathrm{MOR}]=11$, $95 \%$ confidence interval $[\mathrm{CI}]=1 \cdot 3-96)$ and Restaurant $\mathrm{I}(\mathrm{MOR}=5 \cdot 8,95 \% \mathrm{CI}=1 \cdot 2-29)$.

Food-handlers at both restaurants had elevated anti-Vi antibody titres indicative of carrier state. The annual incidence was 5·0/1000 persons. Outbreak-associated costs were $\$ 46000$. Routine or emergency immunization campaigns targeting school-aged children may help prevent or control outbreaks of typhoid fever in endemic disease areas.

\section{INTRODUCTION}

Form the late 1800s to 1951, Salmonella Typhi was the leading cause of salmonella infection in the United States [1]. Improvements in drinking water treatment, safe sewage disposal, shellfish sanitation, and general hygiene of the population have since nearly eliminated $S$. Typhi infections from the United States and other industrialized nations. Today, typhoid fever in the United States is largely associated with international travel to developing countries [2].

In many developing regions of the world, typhoid fever remains a significant cause of morbidity and mortality at a substantial cost to health-care systems.

* Author for correspondence.
Although reliable surveillance data on typhoid fever are sparse, an estimated 16.6 million new infections and 600000 deaths occur globally each year [3]. One region of endemicity is the South and Central Pacific. Several islands, including Tonga [4] and Papua New Guinea [5], have documented high rates of typhoid fever. However, data are limited from other countries in the region.

In 1998-9, an outbreak of typhoid fever occurred in Nauru. This island, located just south of the equator in the Central Pacific Ocean, is the world's smallest republic, covering eight square miles having a population of approximately 10000 persons. Health care is free of charge to native Naurans and employees of the phosphate mines. Medical care is obtained through 
two hospitals. There are no clinics and less than ten practicing physicians at any given time. This report describes the results of an epidemiological investigation conducted in May 1999 to identify the source of the outbreak and prevent further spread. In addition, we present an estimate of the cost of the outbreak and contrast it to the estimated cost of a targeted immunization campaign.

\section{METHODS}

\section{Case finding}

To identify persons with possible $S$. Typhi infection we examined the laboratory bacteriology records at Nauru General Hospital (NGH) and Nauru Phosphate Corporation Hospital, and recorded all persons from 1 October 1998 to 10 May 1999 who had a positive Salmonella spp. or $S$. Typhi culture, or who were screened using the Widal test. In addition, we spoke to the physician in charge of the Typhoid Committee, established in January 1999, to identify additional persons suspected of having typhoid fever. We reviewed medical records to obtain demographic and clinical information on all persons with laboratory-confirmed or clinically suspected typhoid infection and later interviewed these persons.

We defined a clinical case as fever for $\geqslant 3$ days since 1 October 1998, and one or more of the following symptoms: chills, sweats, headache, loss of appetite, malaise, weakness, diarrhoea, constipation, abdominal pain, cough, or vomiting in a resident of Nauru. We defined a confirmed case as a stool, blood, or urine sample yielding $S$. Typhi since 1 October 1998, in a resident of Nauru with illness meeting the clinical case definition.

\section{Case-control studies}

Cases were defined as above. We included only the person with the earliest date of illness onset within a household. With the aid of a translator, persons were administered a written questionnaire that asked about demographic characteristics, illness history and exposures.

In the first case-control study, we included only persons with culture-confirmed illness and two controls matched by age-group and neighborhood. Age matching for controls was as follows: \pm 2 years for patients $<5$ years old \pm 3 years for patients $5-9$ years old; \pm 4 years for patients $10-14$ years old; \pm 5 years for patients $15-19$ years old; and \pm 10 years for patients $\geqslant 20$ years old. Neighborhood controls were selected by skipping the two houses on either side of the case patient's house and interviewing subsequent houses. In the second case-control study, we included persons with culture-confirmed or clinical cases. We selected two controls per culture-confirmed case and one control per clinical case. Controls were identified by randomly selecting household names from the telephone directory and matched using the age categories listed above.

\section{Environmental investigation}

We investigated all 50 restaurants on Nauru at which three or more culture-confirmed patients reported eating. All restaurant employees were queried about illness since 1 October 1998, and requested to provide a blood sample. In addition, employees were asked about the purchase, storage, and preparation of raw fish and shellfish.

\section{Laboratory analysis}

Blood, stool and urine samples were cultured at the NGH laboratory using the Analytical Profile Index (bioMérieux S.A., Marcy-l'Etoile, France). Resistance to antimicrobial agents was measured by disk diffusion. All isolates were tested against ampicillin, chloramphenicol and cotrimoxazole; additional testing was sporadically conducted against other antimicrobial agents. One available strain was confirmed at the Centers for Disease Control and Prevention (CDC). Serum specimens were transported to $\mathrm{CDC}$, where they were tested for anti-Vi antibodies using a passive hemagglutination assay, as previously described [6].

\section{Outbreak costs}

Using 1999 dollars (exchange rate of one Australian $\$=$ US $\$ 0.67$ and one Fijian $\$=$ US\$0.54), we estimated the direct patient-related costs of the outbreak for the 50 patients who met the case definition as well as for 33 additional patients who did not meet the case definition but were treated by clinicians as if they had typhoid fever. The following sources of data were utilized: hospital charges, pharmaceutical costs, and hospital staff salaries obtained from the Nauru Ministry of Health. Epidemiologists and hospital workers estimated the percent of their time spent on typhoid patients during the outbreak. The number of laboratory tests was determined by reviewing lab- 
oratory records. The number of persons treated with antibiotics and with intravenous fluids was determined from hospital records and interviews of patients and medical staff. Missing charts and poor recall most likely resulted in an underestimate of the number of persons treated with antibiotics. We estimated that half the patients hospitalized received intravenous fluids for an average of 7 days.

We estimated the indirect costs among patients caused by lost income by multiplying the following variables: (number of men/women who met the clinical case definition) $\overline{\mathrm{X}}$ (percent of men/women $\geqslant 16$ years in the case-control studies who were employed) $\overline{\mathrm{X}}$ (percent of these men/women who missed work) $\overline{\mathrm{X}}$ (mean number of days of hospitalization for men/women) $\overline{\mathrm{X}}$ (average male/ female income in Nauru). The average male and female income was taken from the Nauru national population census, conducted in 1992.

\section{Estimated cost of a vaccination campaign}

To estimate the cost of implementing a vaccination campaign among schoolchildren aged 5-19 years old we contacted the manufacturer of the typhoid $\mathrm{Vi}$ polysaccharide vaccine (Typhim $\mathrm{Vi}^{\circledR}$, Pasteur Mérieux Sérums \& Vaccins S.A.). According to their pricing for the US federal supply system, the cost of a 10-ml vial with 20 doses is $\$ 347 \cdot 90$ (or $\$ 17 \cdot 40$ per dose). This vaccine is a single injection that can be given to persons 2 years of age and older. Vaccine transportation costs were estimated based on shipment costs from Australia and personnel costs, including one pediatrician and one nurse aide working 15 days, based on salaries from the Nauru Ministry of Health. We determined the number of children aged 5-19 from the 1992 Nauru national population census.

\section{Statistical analysis}

Matched odds ratios, $95 \%$ confidence intervals, and two-tailed $P$-values were computed by using EpiInfo, version 6 (CDC, Atlanta, GA). A $P$-value of $<0.05$ was considered significant. Incidence rates were calculated by using dam from the 1992 Nauru national population census.

\section{RESULTS}

\section{Case finding}

Before the outbreak, the most recent documented cases of typhoid fever had occurred in 1995, when $S$.

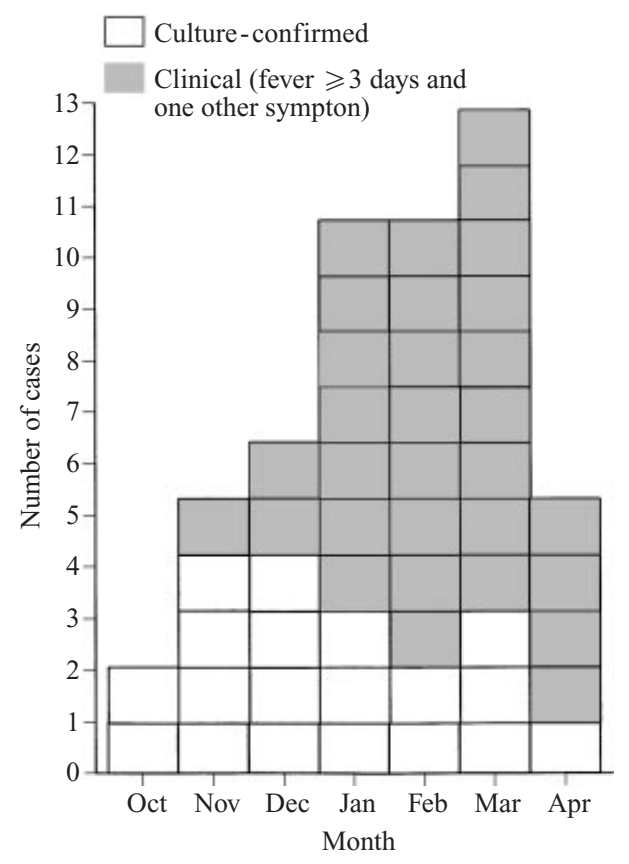

Fig. 1. Cases of typhoid fever by month of onset, Nauru, 1998-9.

Typhi was isolated from three ill persons in one family. We identified 19 persons with laboratoryconfirmed $S$. Typhi infection, all of whom had illness that met the clinical case definition, and 31 others with illness that met the clinical case definition but did not have a specimen tested for $S$ Typhi. Dates of onset ranged from 12 October 1998 to 29 April 1999 (Fig. 1). Among the 50 patients, the median age was 14 years (range 0-54) and 26 (52\%) were men; 32 (64\% of patients were children 5-19 years old.

For the 1-year period from 1 May 1998 to 1 May 1999, the annual incidence of typhoid fever on Nauru was $5 \cdot 0 / 1000$ persons. Rates varied by district, with the highest rates per 1000 population in the northern districts: $35 \cdot 1$ in Anetan, 25.4 in Ewa, 11.1 in Baitsi, and 6.3 in Anabar. Initial cases occurred in the northern districts. The incidence among children aged 5-19 years old was $9 \cdot 4 / 1000$ children.

After fever, the most common signs and symptoms reported were diarrhoea, loss of appetite, headache, chills, and abdominal pain (Table 1). In addition to fever, $96 \%$ of persons had greater than two symptoms and $80 \%$ had greater than three. There were no deaths. Thirty-six persons reported receiving antimicrobial therapy; $32(89 \%)$ of the 36 who recalled the specific antibiotic were treated with chloramphenicol, two with amoxicillin and two with trimethoprim-sulfamethoxazole. Ciprofloxacin was not available in Nauru. Thirty-two $(64 \%)$ patients 
Table 1. Symptoms reported by persons with laboratory-confirmed and clinically defined Salmonella Typhi infection, Nauru 1998-9

\begin{tabular}{ll}
\hline \hline Symptom & No. $(\%)$ \\
\hline Fever & $50(100)$ \\
Diarrhoea & $37(74)$ \\
Loss of appetite & $36(72)$ \\
Headache & $33(66)$ \\
Chills & $31(62)$ \\
Abdominal pain & $30(60)$ \\
Weakness & $28(56)$ \\
Malaise & $28(56)$ \\
Cough & $20(40)$ \\
Sweats & $17(34)$ \\
Constipation & $12(24)$ \\
Rash & $4(8)$ \\
Fever +1 symptom & $50(100)$ \\
Fever $+\geqslant 2$ symptoms & $48(96)$ \\
Fever $+\geqslant 3$ symptoms & $40(80)$ \\
\hline \hline
\end{tabular}

were hospitalized for a median of 17 days (mean 17, range 1-48).

None of the case-patients reported being employed as a food-handler or health-care worker. However, one worked as a laboratory technician and handled outbreak $S$. Typhi specimens before becoming ill. Four households had more than one person ill with typhoid (mean number of ill persons, 4; range 2-9); these families accounted for $17(34 \%)$ of all cases. The mean number of days between illness onset in these households was 18 (range 1-38).

\section{Case-control studies}

We identified no common event or restaurant exposure among case-patients. However, patient recall was greatly limited because of the length of time that had occurred between illness and interview. As a result, we asked about routine activities, including school, work, church, eating out, diet and swimming since 1 October 1998.

In the first case-control study, we enrolled 13 persons with culture-confirmed infection and 26 neighbourhood- and age-matched controls. All 39 persons were Nauruan. There was no difference in the age or sex distribution of patients and controls. Ten $(77 \%)$ of the patients were male compared to 14 $(54 \%)$ of the controls $(P=0 \cdot 3)$. Patients were significantly more likely than controls to have eaten at Restaurant M since 1 October 1998 (matched odds ratio $(\mathrm{MOR})=11,95 \%$ confidence interval $(\mathrm{CI})=$
1.3-96 1). No other variable was associated with illness (Table 2).

Because of concern that neighbourhood controls would be too similar to patients with respect to exposures, a second case-control study was conducted using randomly selected controls. In this study we enrolled 13 persons with confirmed and 14 with clinical cases and 41 randomly chosen, age-matched controls. Besides fever, all patients had greater than two additional symptoms, and $26(96 \%)$ had greater than three additional symptoms. There was no difference in the age of patients and controls, and 18 $(67 \%)$ patients were male compared with $17(41 \%)$ controls $(P=0 \cdot 04)$. Patients were significantly more likely than controls to be Nauruan citizens $(M O R=$ $8 \cdot 7,95 \% \quad \mathrm{CI}=1 \cdot 0-74 \cdot 6$ ) (Table 2). Ninety-three percent of patients in this study (and $94 \%$ of all 50 cases) were Nauruan, compared with $68 \%$ of controls, and an estimated $69 \%$ of the general population of Nauru. Furthermore, patients were more likely than controls to have lived in either Anetan or Ewa districts since 1 October $1998(\mathrm{MOR}=14,95 \% \mathrm{CI}=$ 1.7-117). Patients were significantly more likely than controls to have eaten at the same restaurant identified in the first case-control study, Restaurant M (MOR = undefined, $P=0 \cdot 002$ ). Patients were also significantly more likely to have eaten at a second restaurant, Restaurant I $(\mathrm{MOR}=5 \cdot 8,95 \% \mathrm{CI}=1 \cdot 2-28 \cdot 7)$. Six patients reported eating at Restaurant $\mathrm{M}$ and six at Restaurant I; a total of nine patients ate at either restaurant. Both implicated restaurants are located in the northern district of Ewa.

\section{Environmental investigation}

We identified seven restaurants, including Restaurants $\mathrm{M}$ and $\mathrm{I}$, that were frequented by three or more culture-confirmed patients since 1 October 1998. All interviewed food-handlers denied being ill since 1 October 1998. All restaurants served raw fish. Fish was purchased from fishermen in the district of Aiwo where pipes discharge sewage into the sea. Only one restaurant reported serving shellfish (mussels), which were imported from Australia, purchased frozen from a local store, and served cooked.

\section{Laboratory}

Of the 19 persons with culture-confirmed $S$. Typhi infection, the organism was identified from blood 
Table 2. Proportion of patients and controls exposed to selected variables, matched odds ratio and $95 \%$ CI. Case control study I (patients, $n=13)$ used neighborhood-and age-matched controls $(n=26)$; case-control study II (patients, $n=27)$ used randomly selected, age-matched controls $(n=41)$

\begin{tabular}{|c|c|c|c|}
\hline \multirow[b]{2}{*}{ Exposure } & \multicolumn{2}{|c|}{ Proportion exposed } & \multirow{2}{*}{$\frac{\text { Matched odds ratio }}{(95 \% \text { confidence interval })}$} \\
\hline & $\begin{array}{l}\text { Patients } \\
n(\%)\end{array}$ & $\begin{array}{l}\text { Controls } \\
n(\%)\end{array}$ & \\
\hline \multicolumn{4}{|l|}{ Case-Control Study I } \\
\hline Being Nauruan & $13(100)$ & $26(100)$ & Undefined \\
\hline Living in north district* & $5(38)$ & $10(38)$ & Undefined \\
\hline Eating raw fish & $13(100)$ & $23(88)$ & Undefined, $P=0.54$ \\
\hline Drinking rain catchment & $9(69)$ & $17(65)$ & $1 \cdot 1(0 \cdot 3,4 \cdot 0)$ \\
\hline Restaurant $\mathrm{M}$ & $6(46)$ & $2(8)$ & $11 \cdot 0(1 \cdot 3,96 \cdot 1)$ \\
\hline Restaurant I & $5(38)$ & $5(19)$ & $3 \cdot 5(0 \cdot 6,10 \cdot 5)$ \\
\hline Swimming in ocean & $11(85)$ & $19(73)$ & $2 \cdot 0(0 \cdot 4,11 \cdot 3)$ \\
\hline Swimming near sewage & $5(38)$ & $7(27)$ & $2 \cdot 0(0 \cdot 4,10 \cdot 9)$ \\
\hline \multicolumn{4}{|l|}{ Case-Control Study II } \\
\hline Being Nauruan & $25(93)$ & $28(68)$ & $8 \cdot 7(1 \cdot 0,74 \cdot 6)$ \\
\hline Living in north district* & $8(30)$ & $2(5)$ & $14 \cdot 0(1 \cdot 7,117)$ \\
\hline Eating raw fish & $23(85)$ & $28(68)$ & $7 \cdot 0(0 \cdot 7,67 \cdot 3)$ \\
\hline Drinking rain catchment & $18(67)$ & $21(51)$ & $1 \cdot 9(0 \cdot 7,5 \cdot 8)$ \\
\hline Restaurant M & $6(22)$ & $0(0)$ & Undefined, $P=0.002$ \\
\hline Restaurant I & $6(22)$ & $2(5)$ & $5 \cdot 8(1 \cdot 2,28 \cdot 7)$ \\
\hline Swimming in ocean & $21(78)$ & $28(68)$ & $2 \cdot 2(0 \cdot 6,8 \cdot 1)$ \\
\hline Swimming near sewage & $5(19)$ & $10(24)$ & $0 \cdot 8(0 \cdot 3,2 \cdot 7)$ \\
\hline
\end{tabular}

* North district, Anetan or Ewa.

Table 3. Direct and indirect patient related-costs associated with the Salmonella Typhi outbreak in Nauru, October 1998-April 1999

\begin{tabular}{|c|c|c|c|}
\hline Expense & Total cost & Number affected & $\begin{array}{l}\text { Average cost } \\
\text { per person }\end{array}$ \\
\hline \multicolumn{4}{|l|}{ Direct costs } \\
\hline \multicolumn{4}{|l|}{50 Cases } \\
\hline Physician fees* & $\$ 10965$ & 50 & $\$ 219$ \\
\hline Laboratory costs & $\$ 814$ & 24 & $\$ 34$ \\
\hline Hospitalization costs & $\$ 24183$ & 32 & $\$ 756$ \\
\hline Medication costs & $\$ 81$ & 36 & $\$ 2$ \\
\hline Subtotal & $\$ 36043$ & & \\
\hline \multicolumn{4}{|l|}{33 suspect casest } \\
\hline Laboratory costs & $\$ 281$ & 17 & $\$ 17$ \\
\hline Hospitalization costs & $\$ 3979$ & 6 & $\$ 663$ \\
\hline Medication costs & $\$ 40$ & 15 & $\$ 3$ \\
\hline Subtotal & $\$ 4300$ & & \\
\hline Total direct costs & $\$ 40343$ & 50 & $\$ 807$ \\
\hline \multicolumn{4}{|l|}{ Indirect cost } \\
\hline Lost income of patients & $\$ 5394$ & 13 & $\$ 415$ \\
\hline Total patient related-costs & $\$ 45737$ & 50 & $\$ 915$ \\
\hline
\end{tabular}

* Includes physicians, nurse aide, and laboratory personnel time without benefits.

$\dagger$ Patients who did not meet the case definition.

samples in $9(47 \%)$, from stool in $9(47 \%)$, and from urine in 1 . One isolate, cultured on 12 November 1998, was resistant to ampicillin, cotrimoxazole, gentamicin, and metronidazole; a second isolate, cultured on 16 December 1998, was resistant to ampicillin, augmentin, and cephalothin. The remain- 
ing 17 isolates were susceptible to all antibiotics tested. Unfortunately, all $S$. Typhi strains except one had been discarded before our investigation. This isolate was confirmed at CDC to be $S$. Typhi and was susceptible to amoxicillin-clavulanic acid, ampicillin, ceftriaxone, chloramphenicol, ciprofloxacin, gentamicin, kanamycin, naladixic acid, sulfisoxazole, tetracycline, and trimethoprim-sulfamethoxazole and intermediate to streptomycin. Serum samples were collected from all 15 food-handlers at the 7 restaurants. Two food-handlers had elevated anti-Vi antibody titres, suggestive of being chronic carriers of $S$. Typhi. One food-handler was from Restaurant M (Vi titre, 40) and one from Restaurant I (Vi titre, $.80)$.

\section{Outbreak costs}

The total estimated patient-related costs of the outbreak were $\$ 45737$ (Table 3). These were divided into direct (\$40343) and indirect (\$5394) costs. The majority of the medical costs was attributed to hospitalizations; there were 631 days of hospitalization associated with the outbreak. The average total cost per person infected was \$807. Lost income accounted for $12 \%$ of the total cost of the outbreak.

\section{Estimated cost of a vaccination campaign}

There are approximately 3410 school-aged children 5-19 years old on Nauru. The cost of a single dose of vaccine for each child would be $\$ 59334$. Transportation costs were estimated to be $\$ 500$ and personnel costs, $\$ 1256$. Thus, total estimated costs for a single round of targeted immunization are $\$ 61090$.

\section{DISCUSSION}

This outbreak of typhoid fever was most likely initiated by one or two food-handlers, who were asymptomatic carriers, and was further propagated by subsequent household transmission. The casecontrol studies implicated two restaurants and serologic testing confirmed that two food-handlers were likely to be $S$. Typhi carriers. These two persons were not native Nauruans and history of typhoid fever or travel before the outbreak could not be ascertained. Although the epidemiologic and serologic evidence supports this hypothesis, we were unable to collect stool specimens for $S$. Typhi isolation from the two implicated food-handlers and were therefore unable to link them to the outbreak by molecular subtyping methods.

Transmission of $S$. Typhi occurs through faecally contaminated food or water, especially under poor sanitary conditions. In the two implicated restaurants, the most likely mechanism of contamination was through faecal contamination of food handled by the two employees identified as carriers. We suspect that raw fish or other raw food became contaminated with $S$. Typhi during handling at the restaurant and was then served to the customers. Although it is unusual that two carriers were identified, these results are consistent with the prolonged nature of the outbreak and the apparent diversity of strains suggested by the varying antibiogram. It is likely that the two foodhandlers were not shedding the organism in their stool at the same time, but between the two of them managed to shed over many months. Secondary transmission within four households may have helped prolong and increase the outbreak. As a result of the epidemiologic investigation, one restaurant was temporarily closed and two chronic carriers working in the food industry were identified and treated. Without these actions, continued transmission may have occurred.

The high annual incidence of illness in the two northern districts in Nauru ( $31 / 1000$ persons) is comparable to the rate observed during a typhoid fever outbreak in Dushanbe (18/1000 persons) [7], and is 4-14 times higher than reported endemic (nonoutbreak) incidence rates in other countries. For example, the annual incidence rate per 1000 persons was 8.17 in Papua New Guinea, 8.10 in Indonesia, 6.53 in Nepal, 4.42 in South Africa, and 2.27 in Chile $[5,7]$.

The medical costs, including fixed costs, associated with this outbreak amounted to approximately $\$ 46000$ and hospitalization was $62 \%$ of the total cost. In Nauru, mandatory hospitalization was used to directly observe antimicrobial therapy. Per capita gross national product for low and middle income countries in East Asia and Pacific was US \$970 in 1997 [8]. In 1981, patient-related expenses in a restaurant-associated outbreak of typhoid fever in Texas cost an average of $\$ 4399$ per person [9] compared to $\$ 807$ per person in the Nauru outbreak. In both outbreaks, over $50 \%$ of the costs were associated with hospitalization expenditures, and it is the higher cost of hospitalization in Texas that accounts for the fivefold difference in average costs per person. 
Despite the resolution of this outbreak, there are ongoing risks for typhoid fever in Nauru. Waterborne outbreaks of typhoid fever have the potential to infect large numbers of people [10]. Nauru's desalination plant does not chlorinate water and the risk of waterborne outbreaks of typhoid fever is, therefore, real. Similarly, raw shellfish harvested from sewagecontaminated waters is a well-documented source of typhoid outbreaks [11]. Although raw shellfish were not served at any of the restaurants we investigated, all did serve raw fish. Raw fish could be contaminated with $S$. Typhi directly through contact with sewage, and laboratory studies have demonstrated that $S$. Typhi can survive on certain kinds of fish for over 3 weeks [12]. Finally, there are most likely additional unidentified chronic carriers on Nauru, and perhaps new ones that will arise from this outbreak. Approximately $3 \%$ of persons infected with $S$. Typhi become asymptomatic, chronic carriers. Carriers are a welldocumented source of outbreaks and sporadic infections [11].

One control strategy to consider in future outbreaks of typhoid fever is a vaccination campaign. Historically there has been some reluctance to recommend mass vaccination to control typhoid fever in either an epidemic or endemic setting [13]. However, increases in antimicrobial resistance among $S$. Typhi organisms, and improvements in vaccines have led to increased interest in vaccination as a prevention and control strategy for typhoid fever. To assess the usefulness of such a campaign during an outbreak, we constructed a simple model using data from the current outbreak. If we assume that a vaccination campaign in persons aged 5-19 years was initiated in mid-January after the establishment of the Typhoid Committee, that vaccine efficacy was $70 \%$ at 28 days after immunization (fourfold rise in antibody titres [14]) and nil until that time, then only $8(40 \%)$ of 20 infections in children between 15 January and 1 May 1998, would have been prevented. The cost of eliminating these eight cases was estimated to be $\$ 61090$. However, the cost for treating them was over ninefold cheaper (\$6456). Clearly, vaccination as a control strategy is more costeffective when initiated early in an outbreak when there is a high risk for widespread transmission and disease.

This simplistic model does not account for any prevention of cases acquired through secondary transmission from immunized children, nor for any of the additional benefits that would accrue over the coming years as a result of longer lasting immunity.
Further, the model does not compare various vaccination strategies, such as the use of the oral Ty21a vaccine [15]. An alternative strategy would be the routine immunization of schoolchildren with a typhoid vaccine. This was used to control high rates of endemic typhoid fever in Thailand in the late 1970s and was associated with a sharp decline in typhoid incidence [16]. The future risk of typhoid could also be reduced by treating sewage and disinfecting water supplies. Such public health works have historically controlled typhoid fever in some countries. In any case, as the laboratory-acquired infection in this outbreak illustrates, technicians and microbiologists who process stool specimens that may contain $S$. Typhi should be vaccinated. More data are needed to better define the optimal uses of typhoid immunization for preventing typhoid fever in endemic areas and for controlling typhoid outbreaks.

\section{ACKNOWLEDGEMENTS}

This information was presented at the 37th Annual Meeting of the Infectious Disease Society of America, Philadelphia, PA, November 1999 [abstract no. 591]. The authors thank Dr Moon Shresta, Cindy Limen, Colvin Bill, David Templonvevo, Mervyn Weersinghe, and Calistus Cain of Nauru General Hospital for technical assistance and Drs. Martin Meltzer and Elbasha Elamin of CDC for assistance with the cost analysis.

\section{REFERENCES}

1. Tauxe RV. Salmonella: A postmodern pathogen. J Food Protect 1991; 54: 563-8.

2. Mermin J, Townes J, Gerber M, et al. Typhoid fever in the United States, 1985-1994. Arch Intern Med 1998; 158: 633-8.

3. Pang T, Levine MM, Ivanoff B, Wain J, Finlay BB. Typhoid fever-important issues still remain. Trends Microbial 1998; 6: 131-3.

4. Hirshman JH. Communicable disease in the South Pacific islands, 2. Med J Aust 1976; 2: 794-8.

5. Passey M. The new problem of typhoid fever in Papua New Guinea: how do we deal with it? P N G Med J 1995; 38: 300-4.

6. Nolan CM, Feeley JC, White PC Jr, et al. Evaluation of a new assay for $\mathrm{Vi}$ antibody in chronic carriers of Salmonella Typhi. J Clin Microbial 1980; 12: 22-6.

7. Levine MM. Typhoid fever vaccines. In: Plotkin SA, ed. Vaccines, 3rd ed. Philadelphia: W.B. Saunders Co, 1999: 781-814.

8. The World Bank. 1999 world development indicators. Washington, D.C.: The World Bank, 1999: 14. 
9. Shandera WX, Taylor JP, Betz TG, Blake PA. An analysis of economic costs associated with an outbreak of typhoid fever. Am J Publ Hlth 1985; 75: 71-3.

10. Mermin JH, Villar R, Carpenter $\mathbf{J}$, et al. A massive epidemic of multidrug-resistant typhoid fever in Tajikistan associated with consumption of municipal water. J Infect Dis 1999; 179: 1416-22.

11. Christie AB. Typhoid and paratyphoid fevers. In: Christie AB, ed. Infectious diseases: epidemiology and clinical practice, 4th ed. Vol. 1. Edinburgh: Churchill Livingstone, 1987: 100-64.

12. Mitscherlich E, Marth EH. Microbial survival in the environment: bacteria and rickettsiae important in human and animal health. Berlin: Springer-Verlag, 1984.
13. Tarr PE, Kuppens L, Jones TC, et al. Considerations regarding mass vaccination against typhoid fever as an adjunct to sanitation and public health measures: potential use in an epidemic in Tajikistan. Am J Trop Med Hyg 1999; 61: 163-70.

14. Plotkin SA, Bouveret-Le Cam N. A new typhoid vaccine composed of the Vi capsular polysaccharide. Arch Intern Med 1995; 155: 2293-9.

15. Levine MM, Ferreccio C, Abrego P, et al. Duration of efficacy of Ty21a, attenuated Salmonella Typhi live oral vaccine. Vaccine 1999; 17(Suppl 2): S22-7.

16. Bodhidatta L, Taylor DN, Thisyakorn U, Echeverria P. Control of typhoid fever in Bangkok, Thailand, by annual immunization of schoolchildren with parenteral typhoid vaccine. Rev Infect Dis 1987; 9: 841-5. 